\title{
FLÚOR EN EL AGUA DE CONSUMO Y OTROS FACTORES ASOCIADOS CON LA PREVALENCIA DE FLUOROSIS EN NIÑOS DE 10 A 12 AÑOS DEL CANTÓN COLTA, PROVINCIA CHIMBORAZO
}

\author{
FLUORIDE IN DRINKING WATER AND OTHER FACTORS ASSOCIATED WITH THE \\ PREVALENCE OF FLUOROSIS IN CHILDREN FROM 10 TO 12 YEARS OF THE \\ CANTON COLTA CHIMBORAZO PROVINCE
}

\author{
Esther Yautibug Balla1a, Alexander Cruz Gallegos²a, Ana Armas Vega ${ }^{1 b, 3 c}$
}

\begin{abstract}
RESUMEN
Objetivo: Determinar la prevalencia de fluorosis en los niños de 10 a 12 años del cantón Colta, provincia de Chimborazo, Ecuador, y relacionar su presencia con el porcentaje de flúor presente en el agua de consumo y otros factores asociados. Materiales y métodos: Se realizó un estudio epidemiológico, observacional y descriptivo con una muestra de 219 niños y niñas matriculados en las unidades educativas del cantón, que cumplieron con los criterios de inclusión y exclusión preestablecidos. Ellos fueron evaluados mediante examen clínico-visual con registro fotográfico de sus dientes anteriores superiores e inferiores. Las fotografías obtenidas fueron analizadas por tres personas capacitadas y calibradas en la detección de fluorosis con el índice TF (Thylstrup y Fejerskov). A los padres de los niños se les aplicó una encuesta previamente validada. Resultados: Los resultados mostraron una prevalencia de fluorosis grado 2 y grado 3 , de $36 \%$ y $29 \%$ respectivamente. Se halló una relación directa entre el consumo de bebidas envasadas, ingesta de dentífrico durante el cepillado y la cantidad de dentífrico usado en el cepillo con la presencia de fluorosis, con una diferencia estadísticamente significativa $(p=0,000)$. Conclusión: La prevalencia de fluorosis dental fue de $95 \%$, siendo más acentuados los grados 2 y 3.
\end{abstract}

Palabras claves: Fluorosis dental, prevalencia, flúor. (Fuente: DeCS)

\section{ABSTRACT}

Objective: Determine the prevalence of fluorosis in children of 10 to 12 years of the canton Colta Province Chimborazo and relate its presence with the percentage of fluorine present in drinking water and other associated factors. Materials and Methods: An epidemiological, observational and descriptive study was conducted with a sample of 219 children enrolled in the canton's educational units, which met the pre-established inclusion and exclusion criteria. They were evaluated by visual clinical examination with photographic record of their upper and lower anterior teeth. The photographs obtained were analyzed by three persons qualified and calibrated in the detection of fluorosis index TF (Thylstrup \&amp; Fejerskov). Applied to the parents of the children a previously validated survey. Results: The results showed a prevalence of fluorosis grade 2 and grade 3,36\% and 29\% respectively. It found a direct relationship between the consumption of packaged beverages, intake of dentifrice during brushing and the amount of toothpaste used in the brush with the presence of fluorosis, with a statistically significant difference $(p=0.000)$.Conclusions: The prevalence of dental fluorosis was $95 \%$, grades 2 and 3 being more pronounced.

Key words: Dental Fluorosis, prevalence, fluoride. (Source: MeSH NLM)

\footnotetext{
${ }^{1}$ Universidad Central del Ecuador.

${ }^{2}$ Universidad Tecnológica Equinoccial.

${ }^{3}$ Universidad Sao Paulo, Brasil.

a Odontólogo.

b Profesora de la Carrera de Odontología.

${ }^{\mathrm{c}} \mathrm{PhD}$ y Máster en Operatoria Dental.
}

Este es un artículo Open Access distribuido bajo la licencia Creative Commons Atribución-NoComercialCompartirlgual 4.0

Correspondencia:

Alexander Cruz Gallegos

Correo Electrónico: alexandercruz1993@hotmail.com

Citar como Yautibug Balla E, Cruz Gallegos A, Armas Vega A. Flúor en el agua de consumo y otros factores asociados con la prevalencia de fluorosis en niños de 10 a 12 años del cantón Colta, provincia Chimborazo. KIRU. 2017; 14(2):149 - 156. doi: org/10.24265/kiru.2017.v14n2.7 


\section{INTRODUCCIÓN}

La incorporación del flúor actúa como una barrera de resistencia disminuyendo la solubilidad del esmalte, proporcionando dureza (1), y, por consiguiente, protección dental frente a la caries ${ }^{(2)}$, al reforzar la estructura mineral dental frente al ataque de ácidos. Además, su acción sobre las bacterias cariogénicas inhibe su metabolismo, adhesión y agregación al biofilm dental(3), especialmente cuando es incorporado al empleo del cepillado dental ${ }^{(4)}$; efecto protector determinado con un porcentaje óptimo del ion fluoruro, alrededor de $1 \mathrm{ppm}(1 \mathrm{mg} / \mathrm{l})$, con variaciones de acuerdo con la temperatura del medio ambiente ${ }^{(5)}$. Sin embargo, la ingesta excesiva de flúor puede generar fluorosis dental, también llamada esmalte moteado, que se define como una hipomineralización del esmalte. Ocurre por una interrupción en la formación de la matriz orgánica del esmalte dando la apariencia de opacidad al diente, con grados de afectación dependientes del tiempo y dosis de exposición ${ }^{(6)}$.

El flúor es el elemento más común presente en el agua de consumo ${ }^{(7)}$, con múltiples efectos beneficiosos $^{(8)}$; de ahí la estrategia de incorporarlo en el agua de abastecimiento ${ }^{(9,10)}$; sin embargo, en los últimos años el bombardeo e ingesta prolongada(11) de dentífricos y colutorios y diferentes elementos con elevadas cantidades de flúor ha desencadenado, además de la disminución de la prevalencia de caries $^{(12)}$, alteraciones a nivel dental y esqueletal ${ }^{(13)}$, generando problemas a nivel estético y de función ${ }^{(14)}$.

En 1974, en Ecuador se establece mediante decreto supremo, el programa de fluoración, que inicia la colocación de flúor en el agua. Este programa fue ejecutado en ciertas provincias del país hasta el año 1986. Debido a la baja cobertura del sistema de agua potable, se implementa la fluoración de la sal como medida alternativa en prevención de caries dental ${ }^{(15)}$. Sin embargo, en el año de 1996 el Ministerio de Salud Pública (MSP) realizó un estudio para valorar las concentraciones de flúor presente en el agua proveniente de los páramos de la Cordillera de los Andes y encontró niveles que sobrepasaban los permitidos en el agua potable de distintos cantones. Empero, no se encuentran estudios ejecutados en el cantón Colta, provincia Chimborazo, sobre este tema, pese a la alerta pronunciada por la MSP y la Organización Mundial de la Salud en 1996, que identificó tres provincias y sus respectivas parroquias como zonas con un alto riesgo de fluorosis dental: Chimborazo (Cubujies, Licto, Guamote, San Gerardo),Tungurahua (Picaihua, Totoras, El Rosario, García Moreno, Salasaca) y Cotopaxi (Poaló, Rumipamba R, Muliquindia, Panzaleo, Saquisilí) ${ }^{(15)}$.
El transporte de flúor a partir de los distintos elementos al organismo se produce por vía digestiva (16), almacenándose en el plasma sanguíneo desde el cual se distribuye a los diferentes tejidos. Su absorción constituye un proceso pasivo que acontece en el estómago y a través de las paredes del tracto intestinal en estrecha relación con el ácido gástrico y su presencia de iones $\mathrm{H}+$. Este procedimiento está representado en un $50 \%{ }^{(17,18)}$. La gran afinidad del flúor a los tejidos calcificados explica su depósito en estos tejidos. Su excreción se realiza mediante el riñón y el intestino en tanto que la ingestión de fluoruro es de 0,4 - 0,6 mg en comida y agua por día, y su excreción fecal representa el $8 \%$ de la ingestión total y más del $10 \%$ por vía urinaria ${ }^{(18)}$.

En la saliva, los fluoruros también están presentes en alrededor del 0,01 ppm, constituyéndose el biofilm dental en su principal reservorio ${ }^{(16)}$. La incorporación de flúor al esmalte del diente se produce en diferentes formas de acuerdo con la fase del desarrollo en el que se halle. De esta manera, se puede observar incorporación del flúor durante la formación del diente, cuando es ingerido a través de la vía sistémica hasta llegar al ameloblasto, células que forman el esmalte, siendo el lugar donde produce la síntesis de la matriz proteica para luego calcificarse. En caso de que su consumo se produzca en concentraciones elevadas, se obstruye el metabolismo del ameloblasto produciendo el moteado característico de la fluorosis dental. Eneste proceso, el flúor se asocia con la hidroxiapatita (HAP) constituyendo un tejido más resistente ${ }^{(19)}$, pero con manchas blancas hipomineralizadas con pigmentaciones de color marrón y muy porosas ${ }^{(14)}$, que pueden llegar a la pérdida de continuidad del esmalte del diente de acuerdo con la severidad(20), producto de que el ion ingerido es distribuido hasta alojarse en la matriz del esmalte, lo que reduce el proceso de absorción de proteínas de la matriz que conlleva a la porosidad del esmalte ${ }^{(21)}$.

Buscando detectar el grado de las alteraciones biológicas que el ion flúor produce, Thylstrup y Fejerskov, en $1978^{(22,23)}$, plantearon la clasificación conocida como "Índice TF", que constituye una demostración en secuencia lógica de los diferentes estadios de los defectos causados por la fluorosis considerando la íntima relación de los cambios histopatológicos del esmalte en 10 grados $^{(24,21)}$.

La fluorosis dental está relacionada con diferentes factores. Entre los principales, señalan el hervir el agua de consumo durante 15 minutos, lo que ha desencadenado un aumento de aproximadamente 60 a $70 \%$ de su prevalencia, considerando que el $91 \%$ de la población emplea agua hervida en la leche de fórmula para los recién nacidos y menores de 2 
años. Otro punto lo constituye el consumo de bebidas envasadas que contienen alto contenido de fluoruro $(9,25)$.

Además de los vegetales, el huevo, pollo, pescado así como las sardinas y el salmón contienen concentraciones de flúor entre 6 a $27 \mathrm{ppm}^{(26)}$, observándose también en las áreas volcánicas, en las industrias de aluminio, ladrillos y cerámica que emiten el ion flúor ${ }^{(26)}$, así como en jugos y aguas embotelladas ${ }^{(27)}$.

Con estos antecedentes, el estudio pretendió determinar la prevalencia de fluorosis dental en los niños de 10 a 12 años de edad que asisten regularmente a centros educativos del cantón Colta, provincia de Chimborazo, y relacionarlo con el porcentaje de flúor en el agua de consumo y otros factores asociados al consumo de alimentos y hábitos de higiene oral.

\section{MATERIALES Y MÉTODOS}

Se planteó la ejecución de un estudio epidemiológico, observacional y descriptivo, el cual fue realizado por un solo examinador, el mismo que fue analizado y certificado como viablemente ético por el Comité de Bioética de la Universidad Central del Ecuador. La población de estudio estuvo constituida por 506 niños y niñas de 10 a 12 años de edad, matriculados en las instituciones educativas de las parroquias Columbe y Villa la Unión, escogidas al azar en el cantón Colta. Como criterios de inclusión, los niños participantes debían haber nacido y vivir en las comunidades que pertenecen al cantón Colta y contar con el consentimiento informado, firmado por los padres o representantes, autorizando su participación en el estudio.

Con el permiso respectivo del distrito Colta, Guamote 06D04 del Ministerio de Educación, se procedió a realizar el acercamiento a las instituciones educativas y a los respectivos directores y docentes de estas para socializar el proyecto de investigación en cada institución. Posteriormente, se realizó una reunión de padres de familia con un cronograma de trabajo preestablecido para manifestar la finalidad del estudio y explicar que la participación era libre y voluntaria. Cada padre de familia, previo conocimiento del proyecto, fue solicitado a firmar el documento donde consta el consentimiento informado mediante el cual autoriza la participación de su niño en esta investigación.

Considerando que existen varios factores asociados a la prevalencia de fluorosis, fue aplicada una encuesta previamente validada a los padres de familia que accedieron a llenarla. Esta encuesta constó de 37 preguntas que abarcaban factores sociodemográficos y preguntas relacionadas con factores que contribuyen a la prevalencia de fluorosis, como tipo de agua, alimentos y bebidas que consumen y productos de higiene bucal con alto contenido de fluoruros a los que están expuestos.

Se ejecutó un examen clínico-odontológico a todos los niños cuyos padres voluntariamente permitieron su participación.

Cada participante fue sometido a un registro fotográfico para el cual se utilizaron guantes descartables y un abrebocas para un campo visual más amplio de los dientes anteriores superiores. Con gasa estéril fueron eliminados restos de alimentos y biofilm dental para verificar las características de la fluorosis dental. La toma de registro fotográfico se realizó colocando al niño en un sitio establecido donde la interferencia de luz fue mínima, con una distancia entre el niño y la cámara de $40 \circ 50 \mathrm{~cm}$, estandarizándose la misma intensidad de luz y empleándose una única cámara fotográfica de tubo universal (NIKON D5300) para todo el estudio. El registro fotográfico fue ejecutado por la investigadora principal quien fue debidamente entrenada $y$ capacitada para la toma de dientes anteriores, identificando cada fotografía con un código. Las fotografías obtenidas fueron analizadas por tres personas calibradas en la detección y definición del índice de fluorosis según índice TF.

Para el análisis de las aguas de abastecimiento de (Chiquicas, Secao, Pulucate), fueron recolectados $500 \mathrm{ml}$ de agua en botellas de polietileno. Las tomas fueron realizadas en el mismo lugar en tres periodos de tiempo (en la mañana, al medio día, en la tarde). En cada toma se registró la hora, la fecha y el lugar; luego de lo cual los envases fueron cerrados herméticamente y colados en cooler, en donde se mantuvieron a temperatura ambiente para su posterior traslado al laboratorio de la Facultad de Química de la Universidad Central del Ecuador, donde fue ejecutado el análisis con un espectro fotómetro mediante una solución debidamenteácida, donde los iones de fluoruro forman con el acixotinconpleno glantanos un complejo violeta que determina fotométricamente la concentración de fluoruro. Los análisis, tres por cada toma de agua, permitieron obtener nueve resultados.

\section{RESULTADOS}

El análisis estadístico se hizo mediante software estadístico SPSS V.23. Al tratarse de variables con múltiples opciones, fue aplicada la prueba de ANOVA. Las distribuciones fueron no paramétricas, en este caso realizándose la prueba de Kruskal 
Wallis. Al considerar la prevalencia de los índices de fluorosis en la población de estudio, fue detectado que el TF de grado 2 prevalece con un $36 \%$, el TF de grado 3 se muestra con un $29 \%$; así mismo, se tiene un porcentaje de $18 \%$ de fluorosis de grado 1 , un $5 \%$ de grado 4 , también un $4 \%$ en el grado 5 y un $2 \%$ con el grado 6 , encontrándose un $5 \%$ de niños con dientes totalmente sanos, estableciéndose que un $83 \%$ presenta fluorosis en los grados del 1 al 3 de TF (Figura 1).

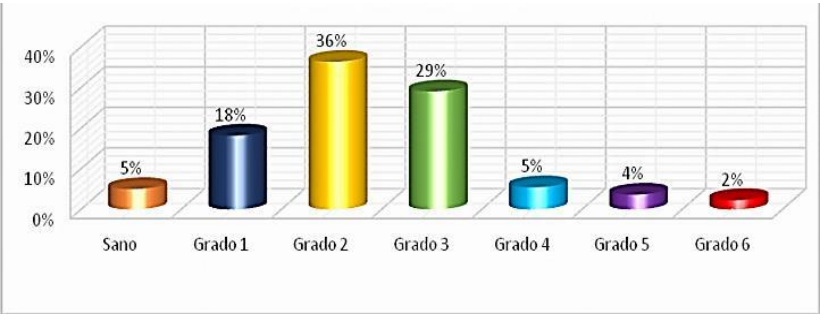

Figura 1. Índice de fluorosis dental en los niños que forman parte de la muestra del cantón Colta.
Con respecto a la relación existente entre el índice de fluorosis relacionado con el género y la zona geográfica, se observa que la zona rural y el género femenino tienen mayor prevalencia de fluorosis en los grados 2 y 3 de TF, con un 19,9\% y 18,4\%, respectivamente, y el grado 1 con un $11,2 \%$; mientras que en la zona rural y en el género masculino muestra mayor prevalencia el grado 2 con un 18,9\%, encontrándose el grado 3 TF con un $10,2 \%$, y el grado 1 con $6,6 \%$. En cuanto a la prevalencia de dientes sin signos de fluorosis se observa un $2,6 \%$ en el género femenino y un $3,1 \%$ en el masculino, encontrándose que los participantes del área urbana de género masculino, tienen mayor prevalencia de fluorosis en los grados 1,2 y 3 con el $16,7 \%, 8,3 \%$ en el grado 4 y $12,5 \%$ en el grado 5 ; mientras que en la zona urbana, el género femenino mostró una prevalencia de fluorosis del $16,7 \%$ solo en el grado 3 deTF (Tabla 1).

Tabla 1. Índice de fluorosis según género y zona geográfica

\begin{tabular}{|c|c|c|c|c|c|c|c|c|c|c|}
\hline Zona geográfica & \multicolumn{2}{|c|}{ Género del niño } & \multicolumn{7}{|c|}{ Índice TF de Fluorosis } & Total \\
\hline \multirow{6}{*}{ Rural } & \multirow{2}{*}{ remenıno } & $F$ & 5 & 22 & 39 & 36 & 5 & 3 & 1 & 111 \\
\hline & & $\%$ & $2,6 \%$ & $11,2 \%$ & $19,9 \%$ & $18,4 \%$ & $2,6 \%$ & $1,5 \%$ & $0,5 \%$ & $56,6 \%$ \\
\hline & \multirow[b]{2}{*}{ Masculino } & M & 6 & 13 & 37 & 20 & 4 & 2 & 3 & 85 \\
\hline & & $\%$ & $3,1 \%$ & $6,6 \%$ & $18,9 \%$ & $10,2 \%$ & $2,0 \%$ & $1,0 \%$ & $1,5 \%$ & $43,4 \%$ \\
\hline & \multirow[t]{2}{*}{ Total } & & 11 & 35 & 76 & 56 & 9 & 5 & 4 & 196 \\
\hline & & $\%$ & $5,6 \%$ & $17,9 \%$ & $38,8 \%$ & $28,6 \%$ & $4,6 \%$ & $2,6 \%$ & $2,0 \%$ & $100,0 \%$ \\
\hline \multirow{6}{*}{ Urbana } & \multirow{2}{*}{ remenıno } & $F$ & & 1 & & $\overline{4}$ & 1 & & & 6 \\
\hline & & $\%$ & & $4,2 \%$ & & $16,7 \%$ & $4,2 \%$ & & & $25,0 \%$ \\
\hline & \multirow{2}{*}{ Masculino } & $\mathbf{M}$ & & 4 & 4 & 4 & 2 & 3 & 1 & 18 \\
\hline & & $\%$ & & $16,7 \%$ & $16,7 \%$ & $16,7 \%$ & $8,3 \%$ & $12,5 \%$ & $4,2 \%$ & $75,0 \%$ \\
\hline & \multirow[t]{2}{*}{ Total } & & & 5 & 4 & 8 & 3 & 3 & 1 & 24 \\
\hline & & $\%$ & & $20,8 \%$ & $16,7 \%$ & $33,3 \%$ & $12,5 \%$ & $12,5 \%$ & $4,2 \%$ & $100,0 \%$ \\
\hline
\end{tabular}

Al relacionar los factores que producen fluorosis dental establecidos en la encuesta con la prevalencia y severidad de la enfermedad en escolares de 10-12 años del cantón Colta y sus parroquias, en la provincia de Chimborazo, considerando el índice de fluorosis y el contacto del participante con una zona industrial mediante la prueba de Kruskal-Wallis, se observó que aquellos niños con mayor grado de fluorosis fueron los que vivieron cerca de una zona industrial ( $p=0,002$ ), lo que significa que existe relación de dependencia entre zona industrial y el índice de fluorosis.
Por otro lado, al relacionar la zona geográfica de residencia del niño con el índice de fluorosis presentado mediante la prueba de Kruskal Wallis, se observó que la variable zona geográfica obtuvo un valor $p=0,041$, lo cual implica que existe una significancia estadística; sin embargo, se evidenció que los niños con grados 4 y 5 TF de fluorosis vivían entre las áreas urbana y rural. 
Al contrastar los valores del índice de fluorosis y el tipo de agua consumida por el niño, se evidenció una presencia de mayores niveles de fluorosis cuando el niño ha consumido agua de pozo y agua filtrada, dando como resultado un valor $p=0,002$, observándose una diferencia estadísticamente significativa entre las variables contrastadas. Esto quiere decir que el tipo de agua consumida por el niño representa un factor de incidencia para la aparecimiento del grado de fluorosis.

Cuando se relacionó el índice de fluorosis y tipo de leche consumida por los niños durante los primeros 4 años de vida, se encontró, mediante la prueba de Kruskal Wallis, que el consumo reportado con mayor frecuencia es la leche del carro repartidor, observándose una diferencia significativa entre estas dos variables; dando como resultado un valor $p=$ 0,049 .

En cuanto a la distribución de las variables índice de fluorosis y cuál bebida envasada es la preferida del niño, se evidencia una tendencia de consumo de jugos, refrescos y té, especialmente en aquellos participantes que en el análisis fotográfico presentaron los grados de fluorosis 5 y 6 , verificándose una diferencia estadísticamente significativa con un valor $p=0,03$, que indica una clara relación del índice de fluorosis con las bebidas preferidas por los niños.

Sin embargo, al considerar el índice de fluorosis y la frecuencia de consumo de estas bebidas envasadas, no se observa ninguna relación ( $p=0,687)$. De la misma manera, al relacionar cuántas veces a la semana el niño consume bebidas envasadas y presenta fluorosis, no se observa relación con la prevalencia de fluorosis dental $(0,199)$.

Al relacionar el índice de fluorosis y el tipo de alimentación del niño, la prueba de Kruskal Wallis ejecutada refiere ausencia de relación $(p=0,741)$. De la misma manera sucede al relacionar el índice de fluorosis y el tipo de sal que consume el niño ( $p=$ $0,226)$.

Por otro lado, al relacionar mediante la prueba de Kruskal Wallis el índice de fluorosis con el consumo involuntario de crema dental durante el inicio del cepillado dental, fue evidenciada una relación con la respuesta afirmativa con todos los grados de fluorosis $(p=0,026)$, indicando una diferencia estadísticamente significativa (Figura 2).

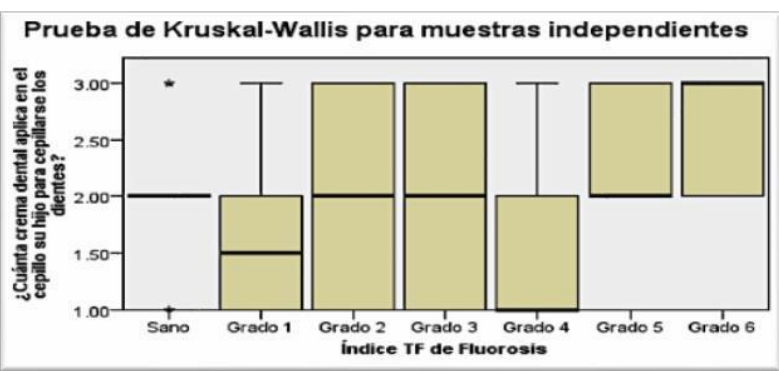

Figura 2. Relación entre el índice de fluorosis y el consumo involuntario de crema dental al inicio del cepillado dental.

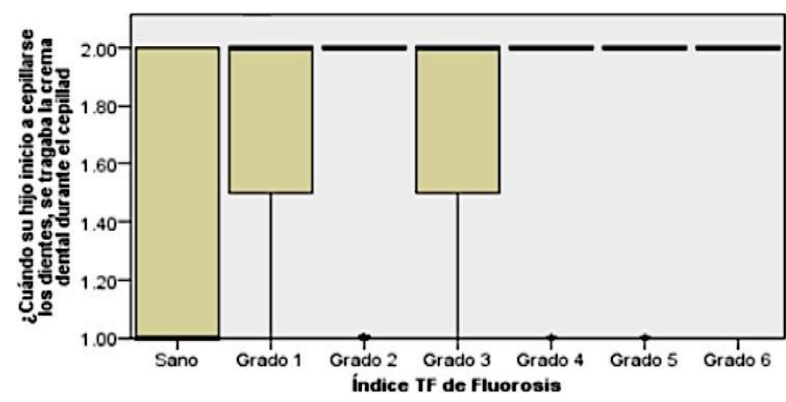

Figura 3. Relación de dependencia entre la variable índice de fluorosis y cuánta crema dental consume.

Al relacionar el índice de fluorosis con la marca de crema dental empleada no se observa ninguna relación $(p=0,842)$. Un resultado similar fue evidenciado al relacionar edad de inicio de cepillado con apoyo de un adulto y grado de fluorosis (valor $p$ $=0,497$ ). De la misma manera, al relacionar el índice de fluorosis y la edad en que el niño inició el cepillado dental por cuenta propia $(p=0,789)$. Resultado similar fue encontrado al relacionar el uso de la crema dental para adultos y el índice de fluorosis ( $p$ $=0,765$ ) y cuántas veces al día cepilla sus dientes y la presencia de fluorosis $(p=0,156)$, pudiéndose afirmar que los hábitos de aseo no inciden en la presencia de un grado de fluorosis.

Al relacionar la variable índice de fluorosis en comparación con la cantidad de crema dental usada en el cepillado por el niño, se verifica que existe una diferencia estadísticamente significativa $(p=0,029)$; es decir, incide en el grado de presencia de fluorosis. No sucede lo mismo al relacionar el índice de fluorosis y el empleo de enjuagues bucales $(p=$ $0,900)$, la edad en que inició su empleo $(p=0,796)$ y la marca de enjuague bucal utilizada $(p=0,679)$, lo que permite asegurar que no existe relación entre estas variables (Figura 3 ). 
Tras los análisis químicos de la concentración de flúor en las tres tomas de agua de abastecimiento examinadas en tres diferentes periodos de tiempo, mañana, medio dia y tarde, los valores obtenidos fueron promediados verificándose que el promedio más alto lo alcanzó el agua tomado de la fuente Chiquicas (un promedio de 1,13 ppm), sin diferencia en cuanto al periodo de recolección, es decir si en la mañana, el mediodía o la tarde.

El análisis de las aguas de las dos fuentes restantes, Pulucate y Secao, presentó valores similares en los dos lugares y en los tres periodos de tiempo (promedio de 0,7 ppm), resultado ideal según las organizaciones de salud mundial (Tabla 2).

Tabla 2. Promedio de la concentración de flúor en el agua, considerando lugar y periodo de evaluación

\begin{tabular}{cccccc}
\hline & \multicolumn{4}{c}{ Periodo de evaluación } \\
\hline $\begin{array}{c}\text { Fuentes de } \\
\text { Abastecimiento }\end{array}$ & Mañana & $\begin{array}{c}\text { Medio } \\
\text { día }\end{array}$ & Tarde & Promedio \\
\cline { 1 - 1 } Secao & 0,70 & 0,70 & 0,70 & $0,70 \mathrm{ppm}$ \\
Pulucate & 0,70 & 0,70 & 0,70 & $0,70 \mathrm{ppm}$ \\
Chiquicas & $\underline{1,13}$ & $\underline{1,11}$ & $\underline{1,16}$ & $\underline{1,13 p p m}$ \\
\hline
\end{tabular}

\section{DISCUSIÓN}

En el presente estudio participaron 219 escolares determinándose en ellos, según el índice de TF, la prevalencia de fluorosis dental en un $95 \%$. Ramírez y otros ${ }^{(28)}$, en el Municipio de Medellín, Colombia, en el 2006, en un total de 330 escolares, refirieron que el $81 \%$ de participantes presentó fluorosis dental. Por otra parte, Pérez et al. ${ }^{(7)}$, en un estudio realizado en México en 355 participantes, encontraron un 94,3\% de prevalencia de fluorosis, teniendo más similitud con nuestro estudio. De igual manera, Mafla et al. ${ }^{(29)}$, en Pasto, Colombia, en 2014 en un total de

599 participantes refirieron que el $94,3 \%$ de escolares presentaron esta alteración.

El estudio ejecutado por el Ministerio de Salud Pública y la Organización Panamericana de la Salud en $1996^{(15)}$ identificó tres provincias y sus respectivas parroquias como zonas con un alto riesgo de fluorosis dental. Entre estas provincias menciona Chimborazo, lo cual motiva a realizar el presente estudio buscando verificar la existencia de la prevalencia de fluorosis dental en niños de 10 a 12 años de edad de las parroquias Columbe y Villa la Unión del cantón Colta, provincia Chimborazo, y su relación con la presencia de factores alimentarios y de higiene oral así como con porcentaje de flúor de las principales fuentes de abastecimiento de agua. Se obtuvieron valores normales: 0,70 ppm en Secao, 0,70 ppm en Pulucate, $1,13 \mathrm{ppm}$ en Chiquicas; valores considerados óptimos según estudios realizados por la OMS ${ }^{(30)}$, los que discrepan de lo referido en el estudio realizado por Ruiz en 1996 (15) quien señala a la zona Guamote, también en la provincia de Chimborazo, como una zona de alta presencia de fluorosis asociada a la cantidad de flúor presente en el agua. Ruiz recomienda ejecutar seguimiento de la concentración de estas aguas, lo que también motivó la ejecución de este estudio al considerar que Guamote constituye un cantón de la provincia de Chimborazo vecino a Colta.

Al analizar los resultados encontrados en este estudio, se pudo verificar que existe prevalencia mayoritaria de fluorosis de grado TF 2 y TF 3 con el $36 \%$ y $29 \%$, respectivamente; seguido de TF 1 con un $18 \%$; así mismo, $5 \%$ de TF 4 y $4 \%$ y $2 \%$ de los grados TF 5 y 6 , respectivamente. Un $5 \%$ de escolares tiene dientes totalmente sanos, siendo este un nivel muy bajo encontrado en esta investigación que establece que la mayoría de los niños (83\%) tiene fluorosis entre el grado TF 1 y TF 3 . Los hallazgos de esta investigación se asemejan con lo encontrado por Galicia et al. ${ }^{(31)}$ quienes refieren en su estudio de 455 escolares de Nezahualcóyotl, Estado de México, en los que se encontró una prevalencia de fluorosis dental con mayor frecuencia de casos en las categorías leve (36 \%) y muy leve (34\%).

En cuanto a la zona geográfica donde habitan los niños de 10 a 12 años del cantón Colta, provincia Chimborazo, encontramos en la zona rural que existe mayor prevalencia en los grados de fluorosis 1,2 y 3 (85\%); mientras que en la zona urbana la fluorosis del grado 2 y 3 se encuentra en un $60 \%$.

Este comportamiento coincide con el estudio de Parra y cols ${ }^{(32)}$ en el 2012, realizado en el Cantón Cuenca, donde los escolares de la zona rural presentaron un aumento del nivel de fluorosis en el $80,7 \%$, mientras que los escolares de la zona urbana se encuentran en el $74 \%$.

Los fluoruros tienen efectos favorables para el ser humano, su introducción en forma tópica y sistémica ha colaborado en la inhibición y prevención de la caries dental ${ }^{(33)}$; sin embargo, cuando su presencia se encuentra en altas concentraciones puede desencadenar una fluorosis dental y esquelética (34), manifestada a nivel dental como una hipoplasia del esmalte.

En este estudio la fluorosis no fue hallada en los grados y frecuencia esperados, encontrándose cierta asociación con el consumo de jugos, refrescos y té, especialmente en aquellos que presentaron grados 5 y 6 , coincidiendo con estudios previos en los cuales la asocian al consumo de bebidas carbonatadas ${ }^{(31}$, 35). 
En relación con la exposición a productos de higiene bucal con alto contenido de fluoruros como factores asociados, este estudio demuestra una concordancia del índice de fluorosis con la variable "si el niño deglute la crema dental durante el cepillado", existiendo una diferencia estadísticamente significativa. Este hecho concuerda con el estudio de Arrieta y otros ${ }^{(36)}$, el cual refiere que los niños menores de seis años por lo general ingieren la pasta dental, afectando este consumo los procesos de mineralización del esmalte, desencadenando la presencia de fluorosis dental ${ }^{(37)}$.

En cuanto a la cantidad de crema dental que utiliza el niño para cepillarse los dientes, se observó una relación significativa con cada uno de los grados de fluorosis, existiendo consumo del total de la pasta que aplica en la escobilla, especialmente en los grados TF5 y TF6, en concordancia con otros estudios ${ }^{(38,2)}$.

Sin embargo, la relación del índice de fluorosis con el uso de enjuagues bucales por el niño no representa un factor a considerar, lo que se explicaría por la ausencia de consumo de estos enjuagues en la población evaluada.

Se observó en las fotografías analizadas la presencia de placa bacteriana que si bien no fue objeto de análisis en este estudio, no fue evidenciada en elevadas cantidades.

\section{CONCLUSIONES}

El porcentaje de flúor en el agua de consumo de las principales fuentes que abastecen al Cantón Colta se encuentra entre 0,70 a $1,13 \mathrm{ppm}$, valores que se encuentran dentro de los parámetros normales según la OMS, descartándose que la causa de fluorosis, sea el flúor presente en el agua de abastecimiento público. Se observó una relación directa entre el consumo de bebidas envasadas, ingesta de dentífrico durante el cepillado y la cantidad de dentífrico usado en el cepillo con la presencia de fluorosis. La prevalencia de fluorosis dental fue de $95 \%$, siendo más acentuados los grados 2 y 3 .

Se recomienda a las autoridades del Ministerio de Salud Publica junto a la comunidad, realizar campañas de prevención de la fluorosis dental y uso de cantidades adecuadas de los productos de higiene bucal que contengan fluoruros y recurrir a la información de esta investigación para dar seguimiento a próximas investigaciones acerca de la fluorosis dental en esta zona y otras donde no existan datos epidemiológicos, con el propósito de contribuir a las comunidades del Cantón Colta.

\section{Contribuciones de autoría:}

EYB y AAV participaron en el diseño de la investigación, revisión de la literatura, recolección de datos, análisis e interpretación de resultados, obtención del financiamiento, redacción y revisión del artículo. ACG: Redacción y revisión crítica del artículo. La redacción final de este artículo tuvo la aprobación de todos los autores.

\section{Fuente de financiamiento:}

Fue financiado por los autores.

\section{Conflicto de intereses:}

No existe un posible conflicto de intereses en este manuscrito. Si existiera, sería declarado en este documento y/o explicado en la página del título.

\section{REFERENCIAS BIBLIOGRÁFICAS}

1. Gonzalez Martínez F, Carmona Arango L, Díaz Caballero A. Percepción de ingesta de flúor a través del cepillado dental en niños de Colombia. Rev. Cubana Estomatol. 2010; 47(3): 266-275.

2 Báez-Quintero LC. Concentración de flúor en cremas dentales y enjuagues bucales para niños vendidos en la ciudad de Bogotá, Colombia. Rev Nac de Odont. 2016; 12(23): 41-48.

3. Hedman J, Sjoman R, Sjostrom I, Twetman S. Fluoride concentration in saliva after consumption of a dinner meal prepared with fluridated salt. Caries Research. 2006; 40(2): 158-162.

4. Ruiz O; Narváez E; Pinto G; Raza X. Estudio del contenido natural de flúor en el agua de consumo humano de los abastecimientos del Ecuador. Quito: Ministerio de Salud Pública/Organización. Panamericana de la Salud; 1996: 1-19.

5. Pérez Patiño T.J, Scherman Leaño R.L, Hernández Gutiérrez R.J, Rizo Curiel G, Hernández Guerrero M.P. Fluorosis dental en niños y flúor en el agua de consumo humano Mexticacán. Medigraphic. 2007; 9(03): 214-219.

6. Núñez Mendieta $H$. Fluorosis dental en niños de localidades del Paraguay con elevado temor de flúor en las aguas de consumo humano. Mem. Inst. Investig. Cienc. Salud. 2011; 09(01): 121-129.

7. De la Cruz Cardoso D, Castillo Chaires I, Arteaga Mejía M, Cervantes Sandoval A, Pinelo Bolaños P. Análisis de la concentración de fluoruro en aguas embotelladas de diferentes entidades federativas de la República Mexicana. Rev. ADM. 2013; 70(2): 81-90.

8. Hurtado Jiménez R, Gardea Torres J. Estimación de la exposición a fluoruros en Los Altos de Jalisco, México. Salud Pública México. 2005; 47(1): 58-63.

9. Gobierno Municipal de Colta. Ubicaciòn geográfica [Online]. 2013 [Citado 2016 diciembre 13]. Disponible en:

http://www.municipiodecolta.gob.ec/municolta/index.p hp/colta/2012-10-01-19-19-46. 
10. Unidad de procesamiento-dirección de estudios analíticos estadísticos - Galo López. www.ecuadorenifras.com. [Online]. 2010 [Citado 2016 octubre 24]. Disponible en: www.ecuadorencifras.com www.inec.gov.ec.

11. Dobarganes Coca A M, López Larquin N, Lima Álvarez M, Calderón Betancourt J, García Mendoza M E. Fluorosis y caries dental en niños de 6 a 12 años. Rev Electrónica Dr. Zoilo E. Marinello Vidaurret. 2015; 40(3): 1-6.

12 Trejo Vázquez R; Bonilla-Petriciolet A. Exposición a fluoruros del agua potable en la ciudad de Aguascalientes, México. Rev. Panam. Salud. 2001 Mayo; 10(2): 108-113.

13. Andrade e Silva SM, Sampaio RC, Oliveira QES, Miranda CB. Microabrasão do esmalte para remoção de manchas de fluorose dentária: relato de caso. Rev Dental Press Estét. 2015; 12(3): 102-109.

14. Miñana V. Flúor y prevención de la caries en la infancia. Rev. Pediatr. Aten Primaria. 2002 septiembre 4(15): 95-123.

15. Chacón Galicia LMFN AGELL. Análisis de la concentración de fluoruro en el agua potable de la delegación Tláhuac, ciudad de México. Rev. Int. Contam. Ambie. 2011; 27(4): 283-289.

16. Soler G. Fluorterapia en Odontología para el niño y e adulto. En: Fluorterapia en Odontología para el niño y el adulto. $3^{a}$. ed. Santiago de Chile: Editorial Adancivia Hermanos y Cia. Ltda; 2001. pp. 24-31.

17. Gutiérrez Rojo, Alma Rosa, Delgado Damaris, Diaz Rogelio. Determinantes de la Salud de la Fluorosis Dental en Nayarit. Oral. 2013; 14(44): 975-978.

18. Salazar M LJC. Prevalencia de fluorosis dental y determinación del grado de severidad en niños de 6 a 15 años en la Florícola Valle Flor ubicada en el Valle de Tumbaco. Odontolnvestigación. 2015: 22-27.

19. Gómez Scarpetta RA, Olaya Pardo M, Barbosa Rivera A, Durán Arismendy L, Vergara Bobadilla H, Rodas Avellaneda CP, et al. Prevalencia de fluorosis dental en infantes de 8 a 12 años de colegios públicos, Villavicencio 2013. Hacia promoc. salud. 2014; 19(1):25-38.

20. Barbería Leache $\mathrm{H}$, Cárdenas E, Suarez M, Maroto M. Fluoruros tópicos: Revisión sobre su toxicidad. Rev. Estomatol. Herediana. 2005; 15(1): 86-92.

21. Martínez A. El problema del flúor en Bélgica: ¿una nueva alarma alimentaria en Europa? Nutricion Hospitalaria. 2002; 17(10): 259-261.

22 Sánchez Rangel C, Neri C, Padilla Ma. Martínez A, Aceves M, Aguilera L. Concentración salival de fluoruro caries y fluorosis dental en escolares de Tacoaleche Zacatecas. Rev. Investig. Cientif. 2008; 4(2): 1-13.
23. López N, Zaragozi E, González L. Efectos de la ingestión prolongada de altas concentraciones de fluoruros.16 de Abril. 2015; 54 (260): 83-94.

24. Hidalgo I, Duque J, Hérnandez F, Zamora J. Fluorosis dental: no solo un problema estético. Rev. Cubana Estomatol. 2007; 44(4): 1-12.

25. Mendoza C. El dilema ético de la fluoración del agua potable. Rev. Méd Chile. 2007; 135: 1487-1493.

26. Harris NO, García-Godoy. Odontología Preventiva Primaria. 2a . ed. Harris NO, Garcia-Godoy, editors. México D.F: El Manual Moderno; 2005.

27. Sousa do Carmo D C; Coelho Alves C; Cavalcante Paulo R; Costa Ribeiro C. Avaliação da fluoretação da água do sistema de abastecimento público na llha de São Luís, Maranhão, Brasil. Ciência \& Saúde Coletiva. 2010; 15(1): 1835-1840.

28. Jones S, Burt B, Petersen P, Lennon M. The effective use of fluorides in Public health. Bull World Health Organ. 2005; 83(9): 670-676.

29. Gasca Reyes. Observación del esmalte dental humano con microscopia electrónica. Rev. Tamé. 2013; 1(3).

30. Gómez F. Histología, Embriología e Ingeniería Tisular Bucodental. 3a․ ed. Ferraris GD, editor. México D.F.: Médica Panamericana; 2009.

31. Negroni M. Microbiología Estomatológica. 2ª ed. Buenos Aires: Médica Panamericana; 2009.

32 Sosa Rosales M. Evolución de la fluoruración como medida para prevenir la caries dental. Revista Cubana Salud Pública. 2003; 29(3): 268-274.

33. Soto Rojas AE, Ureña Cireth JL, Martinez Mier EA. A review of the prevalence of dental fluorosis in Mexico. Revista Panamericana Salud Publica. 2004; 15(1): 918.

34. Olivares Keller D, Arellano Valeria M, Cortés J, Cantín M. Prevalencia y Severidad de Fluorosis Dental y su Asociación con Historia de Caries en Escolares que consumen agua potable fluorurada en Temuco, Chile. Int.J.Odontostomat. 2013; 7(3): 447-454.

35. Espinosa R, Fernández $\mathrm{R}$, Valencia I, Ceja $\mathrm{A}$. Fluorosis dental. Edit. Ripano; 2012

36. Loyola-Rodriguez JP, Pozos-Guillén AJ, HernándezGuerrero JC, Hernández-Sierra JF. Fluorosis en dentición temporal en un área con hidrofluorosis endémica. Salud Pública de México. 2000; 42(3): 194200.

37. Espinosa FR, Valencia HR, Ceja Al. Fluorosis Dental. Etiología, Diagnóstico y Tratamiento. Madrid: Ripano; 2011.

38. Sánchez García S, Pontigo Loyola AP, Heredia Ponce E, Ugalde Arellano JA. Fluorosis dental en adolescentes de tres comunidades del estado de Querétaro. Revista Mexicana de Pediatría. 2004; 71(1): 5-9. 